\title{
Imaging ToF-SIMS and synchrotron-based FT-IR microspectroscopic studies of prostate cancer cell lines
}

\author{
E. Gazi ${ }^{\mathrm{a}}$, N.P. Lockyer ${ }^{\mathrm{a}, *}$, J.C. Vickerman ${ }^{\mathrm{a}}$, P. Gardner ${ }^{\mathrm{a}}$, J. Dwyer ${ }^{\mathrm{a}}$, C.A. Hart ${ }^{\mathrm{b}}$, \\ M.D. Brown ${ }^{\mathrm{b}}$, N.W. Clarke ${ }^{\mathrm{b}}$, J. Miyan ${ }^{\mathrm{c}}$ \\ ${ }^{a}$ Department of Chemistry, Surface Analysis Research Centre, UMIST, P.O. Box 88, Manchester M60 1QD, UK \\ ${ }^{\mathrm{b}}$ CRC Paterson Institute, Christie Hospital NHS Trust, Manchester M20 4BX, UK \\ ${ }^{\mathrm{c}}$ Department of Biomolecular Sciences, UMIST, P.O. Box 88, Manchester M60 1QD, UK
}

Available online 27 April 2004

\begin{abstract}
Imaging ToF-SIMS and synchrotron-based Fourier transform infrared (SR-FT-IR) microspectroscopy have been used to obtain chemical information from individual cells derived from human prostate cancer (CaP). ToF-SIMS imaging of molecular signals characteristic of membrane bound phospholipids are used to elucidate different fracture planes within individual freezefractured $\mathrm{CaP}$ cells. The localisation of $\mathrm{Cu}$ within the cytoplasm of cancer cells is consistent with increased metastatic potential. Line scans across CaP cells using SR-FT-IR microspectroscopy provide complimentary information on the localisation ( $\pm 1 \mu \mathrm{m})$ of lipid and protein domains. This combined analytical approach offers a novel means of characterising individual CaP cells and investigating the biochemical basis of disease progression and metastases.
\end{abstract}

(C) 2004 Elsevier B.V. All rights reserved.

Keywords: Prostate cancer; ToF-SIMS; FT-IR microspectroscopy; Cell lines; Imaging

\section{Introduction}

Prostate cancer $(\mathrm{CaP})$ is the most common cause of cancer related death in males within the UK. Early prognosis is critical for appropriate patient management. Currently prognosis is based on histopathological typing using the Gleason grading system [1]. This approach uses a visual inspection of glandular architectural changes associated with disease progression to indicate the aggressiveness of the tumour at the time of presentation. There is a need for a chemically

\footnotetext{
* Corresponding author. Tel.: +44-161-200-4479; fax: +44-161-200-4439.

E-mail address: nick.lockyer@umist.ac.uk (N.P. Lockyer).
}

based diagnostic approach to grade $\mathrm{CaP}$ tissue samples in a more reliable and reproducible manner. Additionally, biochemical 'markers' are sought, which predict the metastatic potential of the tumour cells to invade other organs - the most common cause of mortality.

Previous work using dynamic SIMS has indicated that ionic imbalances from normal physiological conditions promote tumour growth [2]. We have previously demonstrated that FT-IR microspectroscopic imaging is capable of distinguishing benign from malignant tissue and, with chemometric methods, of providing a diagnostic algorithm for $\mathrm{CaP}$ grading [3]. Here we report the application of imaging static SIMS to the study of human CaP. Additional highresolution spectroscopic imaging is illustrated using 
synchrotron-based Fourier transform infrared (SR-FTIR) microspectroscopy. The combination of these methodologies promises to further our understanding of prostate cancer at a biochemical level.

\section{Experimental}

\subsection{PC-3 cell line preparation for ToF-SIMS}

Human prostate cell carcinoma PC-3 cells (derived $\mathrm{CaP}$ bone metastases) were cultured on silicon or steel wafers in Ham's 12 nutrient medium (PAA Laboratories, Austria) at $37^{\circ} \mathrm{C}$. To prevent distortion of cell morphology polymer microspheres ( $4.5 \mu \mathrm{m}$ diameter) were added to samples destined for fracture prior to adding a second wafer to complete a 'sandwich'. Samples were fast frozen in liquid propane and fractured under liquid nitrogen. This frozen sandwich methodology has previously been used to prepare cellular samples for dynamic or static SIMS analysis [4,5]. Fractured samples were freeze-dried overnight prior to analysis at room temperature. A second set of samples were fast frozen and freeze-dried immediately after culture, providing intact cells for analysis.

\subsection{ToF-SIMS analysis}

ToF-SIMS studies were carried out on a "BioToFSIMS" instrument developed in collaboration with Penn State University and Kore Technology Ltd. and described by Braun et al. [6]. Analysis was performed with a $15 \mathrm{keV} \mathrm{Ga}^{+}$primary ion source (Ionoptika Ltd.) (1.7 nA continuous current). Secondary ions were analysed in a reflectron mass spectrometer and detected with a dual microchannel plate assembly operating with $20 \mathrm{kV}$ post-acceleration. Flight times were recorded with a $1 \mathrm{~ns}$ time-todigital converter.

\subsection{LNCaP cell line preparation for SR-FT-IR}

LNCaP cells (derived from CaP lymph node metastases) were cultured on non-toxic MirrIR reflectance plates (Kevley Technologies, $\mathrm{OH}$, USA) at $37{ }^{\circ} \mathrm{C}$. Cell lines were critical point dried (CPD) in $\mathrm{CO}_{2}$ leaving the cells in a well-preserved state for FT-IR analysis.

\subsection{SR-FT-IR analysis}

To achieve spectral differentiation at the single-cell level with sufficient signal to noise requires the use of intense synchrotron radiation. IR spectral line-scans were collected using synchrotron-based FT-IR (SRFT-IR) at the Daresbury Laboratory (UK). Spectra were recorded in reflectance mode with $16 \mathrm{~cm}^{-1}$ resolution and $2 \mu \mathrm{m}$ step size using a Nicolet Nicplan 860 IR spectrometer.

\section{Results and discussion}

\subsection{ToF-SIMS analysis}

ToF-SIMS images of fractured and unfractured PC3 cells are shown in Fig. 1. Elemental ion imaging of the fractured and unfractured cells (Fig. 1) confirm good preservation of these cells by the fast-freezing method, showing relatively low levels of intracellular $\mathrm{Na}^{+}$and relatively high levels of intracellular $\mathrm{K}^{+}$. In healthy cells the $\mathrm{Na}^{+}-\mathrm{K}^{+}$pumps in the plasma membrane maintains an intercellular $\mathrm{K}^{+}: \mathrm{Na}^{+}$concentration of approximately 10:1.

A wide range of molecular ions are observed from both fractured and unfractured cells under static analysis conditions. Molecular fragments characteristic of different fracture planes have previously been identified in ToF-SIMS analysis of frozen-hydrated cells and liposomes [5,7]. Phospholipids such as phosphatidylcholine (PC) and phosphatidylethanolamine (PE) are common components of cellular membranes. The levels of PC (indicated by the PC headgroup $\mathrm{C}_{5} \mathrm{H}_{15} \mathrm{NPO}_{4}{ }^{+}$at $\mathrm{m} / \mathrm{z}[184]^{+}$) from within the fractured cells were observed to be relatively low (not shown) and arise due to its presence in membrane components of cytoplasmic organelles. In contrast, relatively high levels of PC are found localised in unfractured cells (Fig. 1c). These observations are consistent with the fact that $\mathrm{PC}$ is the most abundant phospholipid on the outer leaflet of the cell membrane. Unfractured cells also exhibit high yields of hydrocarbon fragments such as $\mathrm{C}_{4} \mathrm{H}_{7}{ }^{+}$at $\mathrm{m} / \mathrm{z}[55]^{+}$due to the tail-groups of the membrane lipid bilayer (Fig. 1d).

The ToF-SIMS spectra, extracted from pixels corresponding to the fracture cell and unfractured cells, are shown in Fig. 2a and b, respectively. We find that 


\section{Unfractured cells}

(a) $\mathrm{Na}^{+}$

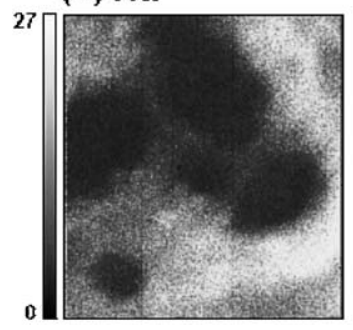

\section{Fractured cells}

(e) $\mathrm{Na}^{+}$

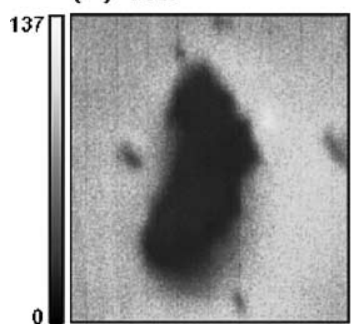

(b) $\mathrm{K}^{+}$

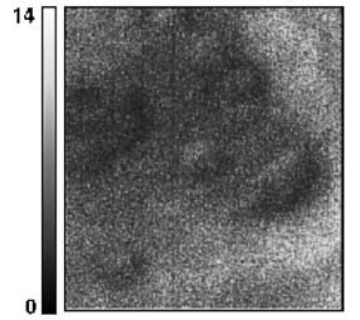

(c) $m / z[184]^{+}$

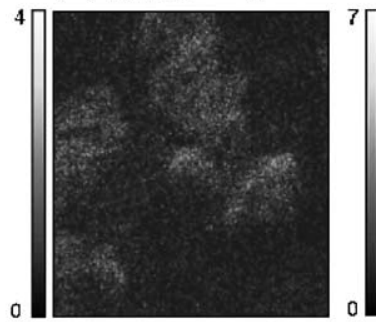

(d) $\mathrm{C}_{4} \mathrm{H}_{7}+$

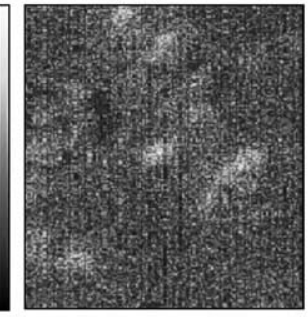

(f) $\mathrm{K}^{+}$

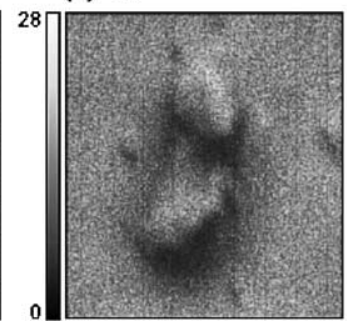

(g) $m / z[168]^{+}$

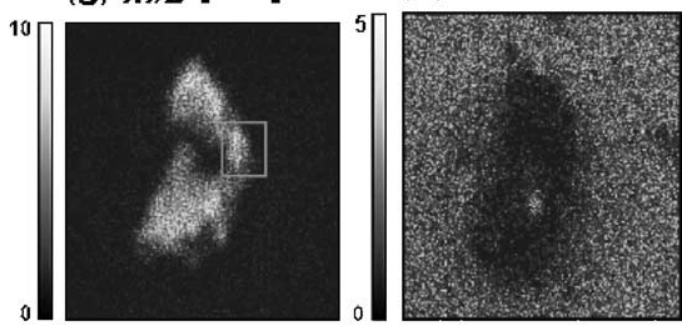

Fig. 1. Positive ion ToF-SIMS images of freeze-dried PC-3 cells: (a)-(d) clustered and individual unfractured cells, $150 \mu \mathrm{m}$ f.o.v.; (e)-(h) two freeze-fractured cells, $100 \mu \mathrm{m}$ f.o.v. Ions imaged and intensity scales are displayed next to images.

the characteristic PC fragment at $\mathrm{m} / z[184]^{+}$is barely detectable in the fractured cells in contrast to the intact cell. However, the peak at $\mathrm{m} / \mathrm{z}[168]^{+}$in the fractured cells has significantly risen in intensity relative to the unfractured cell spectrum. We suggest that the peaks at $m / z[168]^{+}$and $m / z[124]^{+}$are characteristic fragments of the PE head group. Lipid composition of the inner and outer leaflet of the lipid bilayer differ significantly in different cell types and phospholipid molecules that contain a terminal primary amino group (PE and phosphatidylserine) locate preferentially within the inner cell membrane [8]. In light of this fact and in correlation with the $\mathrm{K}^{+}$ion image shown in Fig. If we infer that the fractured cells exhibit two distinct cellular surfaces. Whilst the majority of the exposed cell surfaces are cytoplasm, the region denoted by the white box (Fig. 1g) shows low levels of cytoplasmic $\mathrm{K}^{+}$and high levels of PE fragments. We suggest that these regions may represent inner-leaflet material derived from either plasma
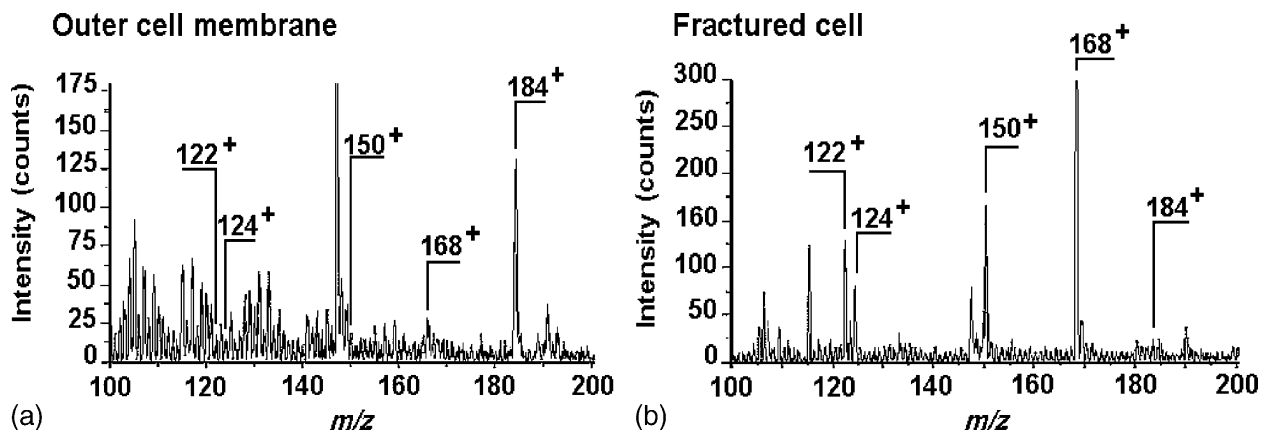

Fig. 2. Positive ion ToF-SIMS spectrum of pixels corresponding to (a) unfractured PC-3 cells and (b) fractured PC-3 cells. The intensity scale is expanded to show low emitting secondary ions. 
membrane in contact with cytoplasm or cytoplasmic organelles.

An interesting feature observed within the cytoplasm of the fractured PC-3 cell is the localisation of $\mathrm{Cu}^{+}$(Fig. 1h). Additionally, we observe low $\mathrm{Cu}$ signals at the membrane-exposed faces (areas with no $\mathrm{K}^{+}$localisation surrounding the cells). It has been reported that epithelial cells possess distinct mechanisms of metal uptake at the apical and basolateral membranes [9]. In the cell, $\mathrm{Cu}$ is an essential cofactor for many enzymatic activities and has been associated with increasing the risk of metastasis for cancer cells by mediating angiogenesis [10]. Cu may be incorporated into the PC-3 cells from the nutrient medium, Ham's F12, which contains $\mathrm{CuSO}_{4}$.

\subsection{SR-FT-IR analysis}

ToF-SIMS can provide information regarding specific lipid and trace inorganic ion compositions from within different sections of a freeze-fractured cell. However, due to excessive fragmentation of biomolecules observed in the ToF-SIMS spectrum, it is difficult to assign secondary ion peaks to protein species. Therefore, we have utilised SR-FT-IR to investigate protein localisation, as well as lipid content, within intact $\mathrm{CaP}$ cells.

A line map across two CaP cells was taken at $2 \mu \mathrm{m}$ step size using a sampling aperture of $5 \mu \mathrm{m} \times 5 \mu \mathrm{m}$ (Fig. 3). At a distance of $6 \mu \mathrm{m}$ from the start of the line map (S), we see that absorption of the protein amide I band is higher relative to the substrate indicating the detection of the cell and conforms to measurements in the optical image. It is interesting to note that the protein absorption at $6-10 \mu \mathrm{m}$ is higher than any other part of cell 1. It is known that epithelial cells migrate with a leading edge called lamellipodia [11], which are rich in protein actin filaments. We suggest that this structure exists at the boundary of the cell, extending $4 \mu \mathrm{m}$ into the cell, beyond which cytoplasmic material housing the cell organelles is present. This may also be visualised in the optical image by a slight change in contrast.

At a distance of 18-32 $\mu \mathrm{m}$ we observe high lipid absorption, relative to cytoplasm $(32-41 \mu \mathrm{m})$, which is attributed to IR radiation transmitting through the nuclear double membrane in addition to the cell plasma membrane and membranous components of
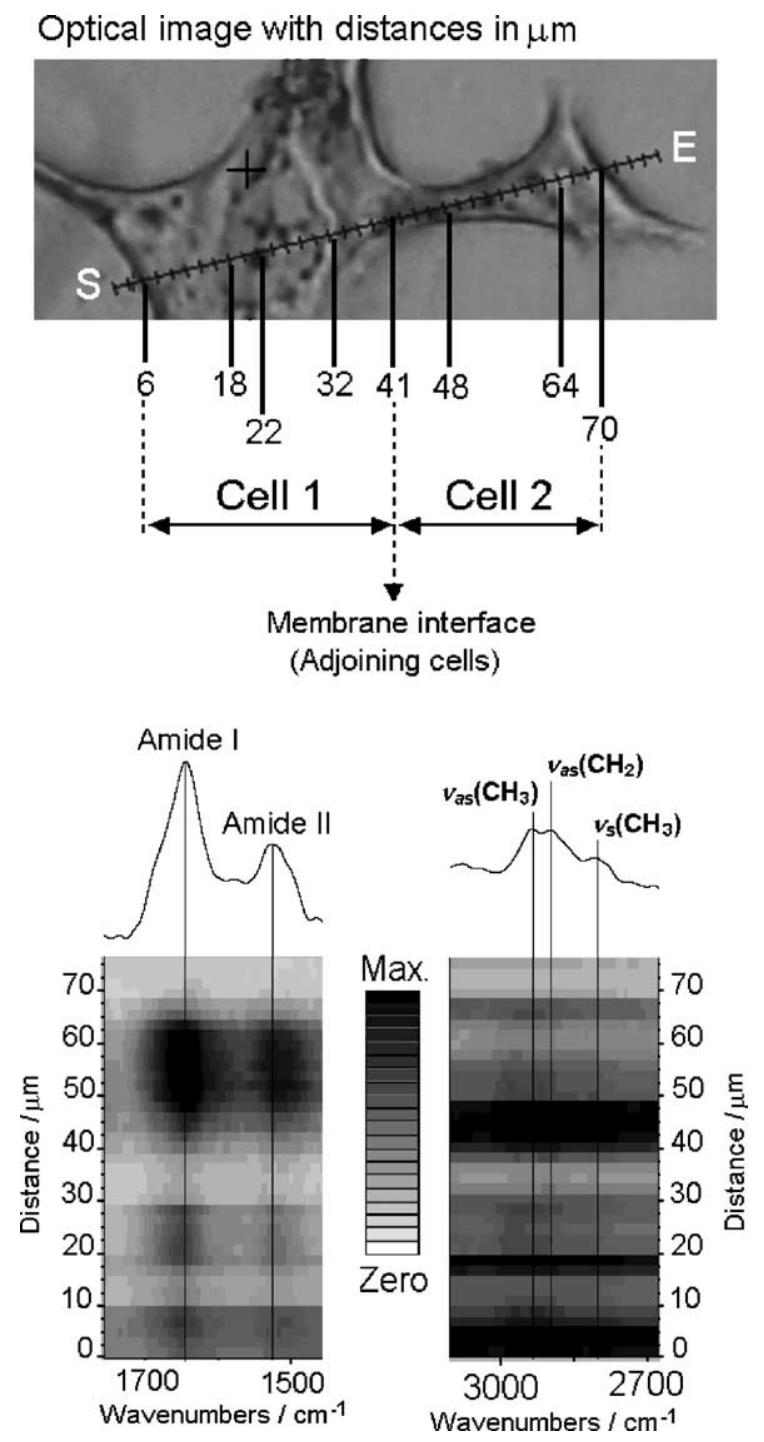

Fig. 3. Optical image of two prostate cancer cells. Line map was taken at $2 \mu \mathrm{m}$ step size with an aperture of $5 \mu \mathrm{m} \times 5 \mu \mathrm{m}$. S and $\mathrm{E}$ on the optical image designate the start and end points where from spectra were recorded. Measurements designated (in $\mu \mathrm{m}$ ) are used to investigate spatial localisation of different biochemical components of the cell (see text). The corresponding intensity contour plot is presented for the line map for the protein amide I and II regions $\left(1500-1475 \mathrm{~cm}^{-1}\right)$ and lipid region $\left(3000-2700 \mathrm{~cm}^{-1}\right)$.

cell organelles. We observe high amide I absorption at $22 \mu \mathrm{m}$ due to the histone protein of nucleosomes. At $41 \mu \mathrm{m}$ we observe high lipid absorption, which may be attributed to the membrane interface between the two adjoining cells. This is accompanied by high 
protein absorption, suggesting the presence of lamellipodia where cells are migrating away from each other.

The amide I band stops detecting the nucleus at $64 \mu \mathrm{m}$ with a corresponding drop in intensity, which conforms to measurements in the optical image (at $62 \mu \mathrm{m}$ a nucleosome is present; at $64 \mu \mathrm{m}$ perinuclear space and nuclear membrane is present). The boundary of cell two is detected at $70 \mu \mathrm{m}$ by low amide I and lipid absorptions.

\section{Conclusions}

Static SIMS imaging of elemental and molecular ions provides the basis for a valuable insight into specific biochemical compositions of the PC-3 cell line. The freeze-fracture process may expose different intracellular domains within individual cells. These domains may be identified using imaging ToF-SIMS. The FT-IR data reported here demonstrates biochemical localisations of lipid and protein domains-other regions of the IR spectrum can provide information on carbohydrate, phosphate and nucleic acid domains. ToF-SIMS and FT-IR spectroscopy, in combination, can potentially be applied to monitor phenotypic variations upon exposure to different stimuli. Such analyses may potentially provide the molecular biologist with a focused line of research in targeting specific ion channels and biomolecules involved in cancer progression.

\section{Acknowledgements}

We acknowledge the UK Engineering and Physical Sciences Research Council (EPSRC) for financial support. We thank Mr. Emrys Jones (UMIST) and Dr. Mark Tobin (Daresbury Laboratory) for assistance in collecting ToF-SIMS data and FT-IR data, respectively.

\section{References}

[1] D.F. Gleason, Histologic grading and clinical staging of prostatic carcinoma, in: M. Tannenbaum (Ed.), Urological Pathology - The Prostate, Lee and Febiger, Philadelphia, 1977, pp. 171-197.

[2] S. Chandra, Appl. Surf. Sci. 203/204 (2003) 679.

[3] E. Gazi, J. Dwyer, P. Gardner, A. Ghanbari-Siakhali, A.P. Wade, J. Myan, N.P. Lockyer, J.C. Vickerman, N.W. Clarke, J.H. Shanks, C. Hart, M. Brown, J. Pathol. 201 (2003) 99.

[4] S. Chandra, G.H. Morrison, Biol. Cell 74 (1992) 31.

[5] T.P. Roddy, D.M.S.G. Ostrowski, N. Winograd, D.M. Cannon Jr., A.G. Ewing, Anal. Chem. 74 (2002) 4020.

[6] R.M. Braun, P. Blenkinsopp, S.J. Mullock, C. Corlett, K.F. Willey, J.C. Vickerman, N. Winograd, Rapid Commun. Mass Spectrom. 12 (1998) 1246.

[7] B. Cliff, N. Lockyer, H. Jungnickel, G. Stephens, J.C Vickerman, Rapid Commun. Mass Spectrom. 17 (2003) 2163.

[8] D. Dalake, W. Huestis, J. Cell Biol. 108 (1989) 1375.

[9] C.J. Matthews, E.J. Thomas, C.P.F. Redfern, B.H. Hist, Hum. Reprod. 8 (1993) 1570.

[10] Q. Pan, et al., Cancer Res. 62 (2002) 4854.

[11] D.M. Prescott (Ed.), Cells: Principles of Molecular Structure and Function, Jones and Bartlett, USA, 1988, p. 484. 